\title{
Distinctive Characteristic Features of Intramedullary Hemangiopericytomas
}

\author{
Cezmi Çağrı Türk ${ }^{1}$, Niyazi Nefi Kara ${ }^{1}$, Dinç Süren ${ }^{2}$, Çağatay Özdöl ${ }^{1}$, Tolga Gediz ${ }^{1}$, Sevim Yıldız ${ }^{3}$ \\ ${ }^{1}$ Department of Neurosurgery, Ministry of Health, Antalya Education and Research Hospital, Antalya, Turkey \\ ${ }^{2}$ Department of Pathology, Ministry of Health, Antalya Education and Research Hospital, Antalya, Turkey \\ ${ }^{3}$ Department of Radiology, Ministry of Health, Antalya Education and Research Hospital, Antalya, Turkey
}

Study Design: The retrospective analysis of intramedullary hemangiopericytomas (HPCs) was performed, and the entity was discussed in accordance with the literature findings.

Purpose: This study aimed at defining distinctive characteristic features of intramedullary HPC with respect to surgical approach and prognosis.

Overview of Literature: Intramedullary HPCs are extremely rare tumors. They originate from capillary pericytes, supposedly follow the vessels over the spinal cord, and infiltrate deep into the spinal cord without a distinct plane. Their treatments and prognosis are not well-defined in the literature.

Methods: Our database was retrospectively reviewed for the cases of HPCs. Later on, a literature search was performed to reveal all reported cases of intramedullary HPCs. The following key words were searched in PubMed databases: "hemangiopericytoma and intramedullary," "hemangiopericytoma and spine (spinal) and intradural," and "hemangiopericytoma and spinal cord." The articles were reviewed for patients' demographics features, imaging characteristics, tumor-specific factors (surgical technique, pathological descriptions, and world health organization grades), and postoperative course and prognosis (adjuvant therapies, recurrences, complications, and mortalities).

Results: A total of seven patients (three male and four female) was reached, with their ages ranging from 15 to 80 years (mean, 32.5 years). The tumors were located majorly in thoracic region $(5 / 7,71.4 \%)$, and only two cases were in the cervical region $(2 / 7,28.6 \%)$. All tumors were completely removed, and only two cases received radiotherapy. No recurrence was reported.

Conclusions: Complete resection of the intramedullary HPCs seems to be the best management strategy for long-term and recurrence-free survival and in alleviating further need for radiotherapy.

Keywords: Spine; Intradural; Intramedullary; Hemangiopericytoma; Surgery

\section{Introduction}

Hemangiopericytomas (HPCs) are rare tumors, which is thought to comprise $2.4 \%$ of meningeal tumors and less than $1 \%$ of all central nervous system tumors [1]. In 1942,
Stout and Murray [2] described a vascular tumor arising from pericytes, which are contractile cells around the capillaries. They suggested a distinctive name, "hemangiopericytoma." These tumors were accepted at first as angioblastic variants of meningiomas; however, in 1993,

Received Dec 22, 2014; Revised Jan 5, 2015; Accepted Jan 19, 2015

Corresponding author: Cezmi Çağrı Türk

Antalya Eğitim ve Araştırma Hastanesi, Beyin Cerrahisi Kliniği, Varlık Mah, Muratpaşa, Antalya, 07050, Turkey

Tel: +90-53323425 93, Fax: +90-242 24944 62, E-mail: drcezmiturk@gmail.com 
the World Health Organization (WHO) recognized these tumors as distinct clinicopathological entities $[3,4]$.

A previous literature review in 2009 indicated only 10 cases of intradural involvement in the total of 52 spinal cases [5]. Intramedullary involvement or invasion of these tumors is extremely rare, and the first case was reported in 1990 by Ujiie et al. [6].

The following study aimed at reviewing the literature for intramedullary involvement $\backslash$ invasion of HPC and their characteristics features.

\section{Materials and Methods}

\section{Search strategy}

The National Library of Medicine's PubMed database was searched for the following terms: "hemangiopericytoma and intramedullary," "hemangiopericytoma and spine (spinal) and intradural," and "hemangiopericytoma and spinal cord." The first article was dated to 1990 [6]. The articles concentrating on intramedullary HPC, with the clinical features, surgical technique, resection rates, and prognosis, were targeted. The articles publicized in languages other than English and HPCs without intramedullary involvement were excluded from this study.

\section{Data collection}

The articles were reviewed for patients' demographics (age, sex, symptoms, and durations), imaging characteristics (magnetic resonance [MR] findings, location, and preoperative diagnosis and differentials), tumor-specific factors (surgical technique, pathological descriptions, and WHO grades), and postoperative course and prognosis (adjuvant therapies, recurrences, complications, and mortalities).

\section{Results}

Our search reached a total of 233 articles with aforementioned the key words. A total of six articles was identified eligible to be included in the review. Most of the other articles were eliminated, because they consisted of the cases other than intramedullary HPCs. Besides, the publications in languages other than English were excluded, since the characteristic features of the tumor could not be specified. Among these articles, one reported by Ujiie et al. [6] could not been taken into consideration due to language difference (Japanese). Moreover, another article could not be included in this study, because we could not confirm the exact location of the tumor from the figures in the article, although it was reported to be in the intramedullary region at L4-S1 level [7]. Therefore, five cases from four articles in the literature search and two case of our series (Figs. 1-5) were considered, which makes it a
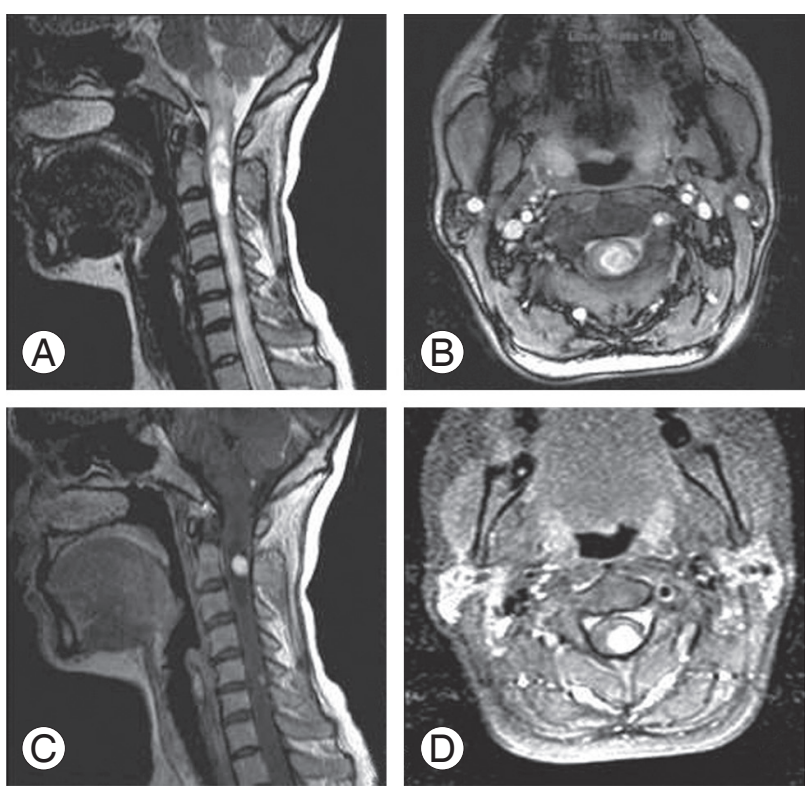

Fig. 1. Preoperative magnetic resonance imaging (MRI) scans of case 1. (A) and (B) show T2-weighted sagittal and axial MR images demonstrating intramedullary mass lesion and surrounding syrinx cavity at C2 level, expanding to the spinal cord. (C) and (D) show post contrast T1weighted sagittal and axial MR images with prominently enhancing mass lesion in the spinal cord.
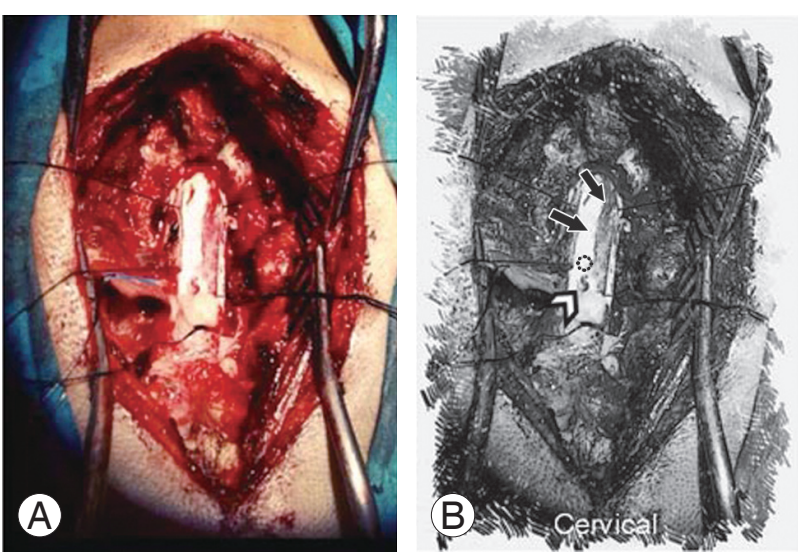

Fig. 2. (A, B) Intraoperative picture following dural opening is shown. (B) Black arrows indicate intermingled capillaries located over the spinal cord. The arrow head indicates the prominent feeder artery supplying the tumor from below. The circumferential dots show the approximate location of the tumor. 

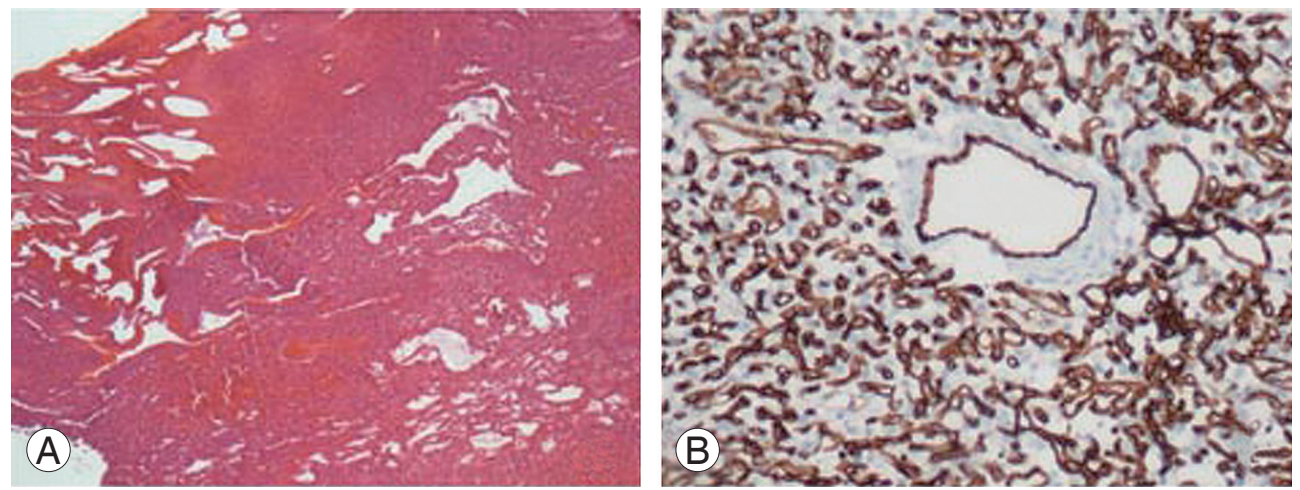

Fig. 3. (A) Vascular tumor adherent to spinal dura mater $(H \& E, \times 40)$. (B) Positive CD34 staining in endothelium of thick-walled vessels and stromal cells $($ CD34, $\times 200)$.
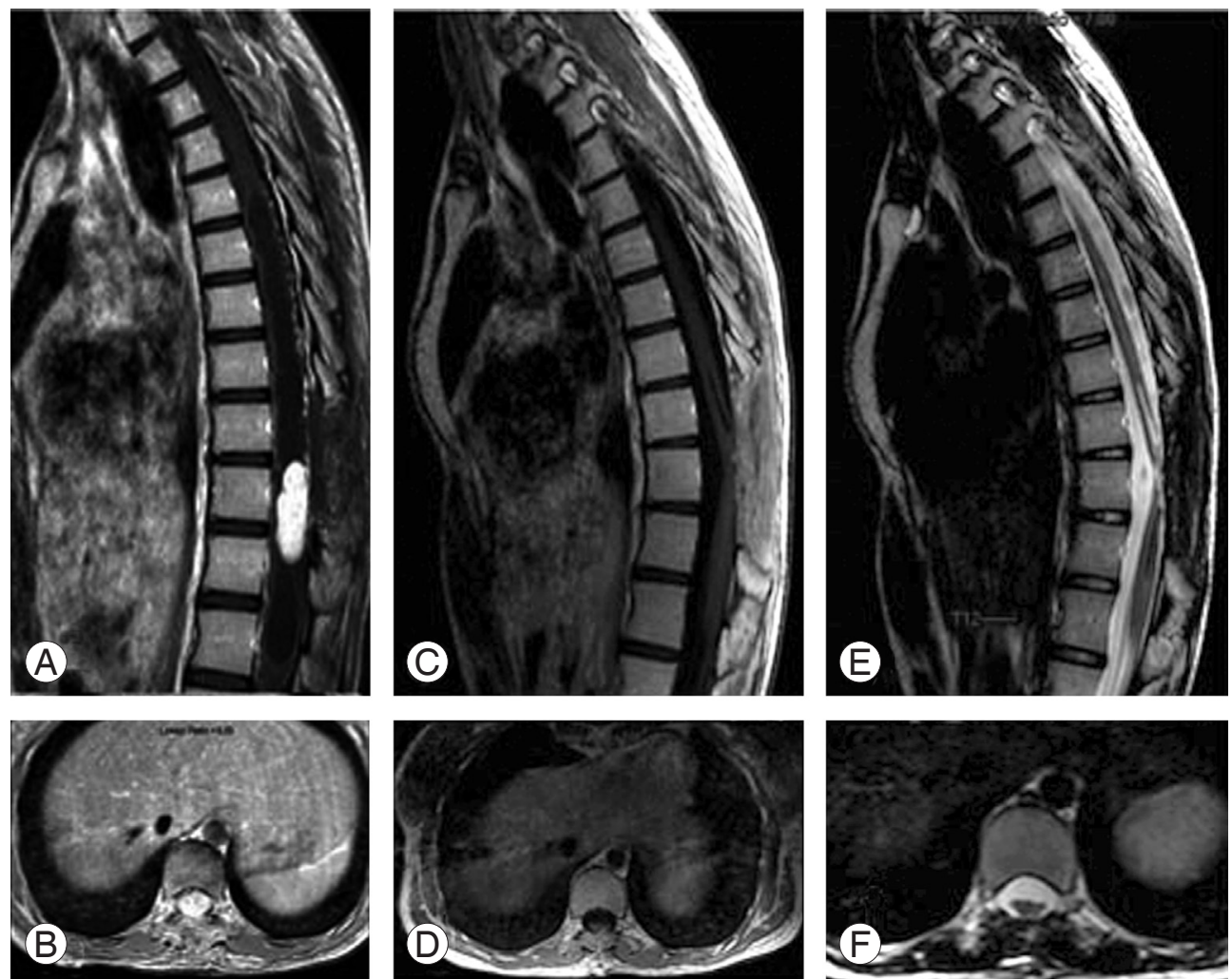

Fig. 4. Preoperative and postoperative magnetic resonance imaging scans are shown for the case number 2. (A) and (B) show preoperative post contrast T1-weighted sagittal and axial images with highly enhancing mass lesion and syrinx cavity expanding superiorly and inferiorly in thoracic spinal cord. (C) and (D) show postoperative post contrast images indicating no residual tumor. (E) and (F) show postoperative T2-weighted images demonstrating spinal cord thinning and decrease in the syrinx cavity.

total of seven cases included in the review (Table 1) $[5,8-$ 10].

\section{Patient characteristics}

There were three male and four female patients, aging between 15 to 80 years (mean, 32.5 years). The major complaint was progressive weakness in the lower extremities lasting approximately 2-4 months, in almost all of the cases. The search indicated that cervical HPCs were mostly presented with ataxia and unsteadiness feeling. 

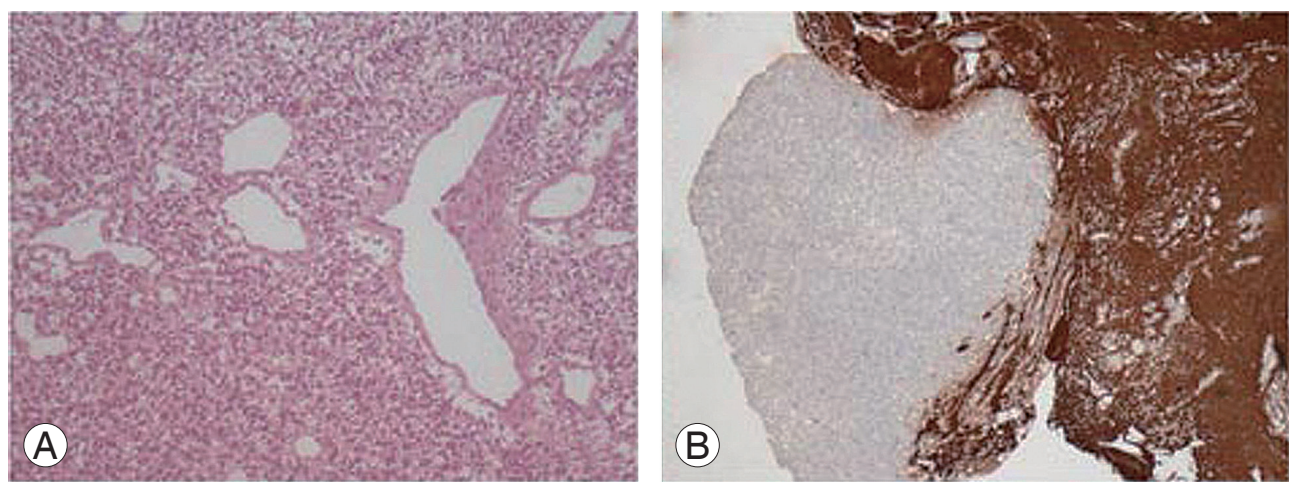

Fig. 5. (A) Stag-horn vascular structures and neoplastic stromal cells around the slit shaped vascular structures $(H \& E, \times 100)$ are shown. (B) Repellent infiltration to glial tissue can be discriminated with GFAP (glial fibrillary acidic protein, $\times 40$ ).

Table 1. Review of the literature for intramedullary involvement of hemangiopericytoma regarding details for demographics, localization, level, surgical treatment and recurrences of the lesions

\begin{tabular}{|c|c|c|c|c|c|c|c|c|}
\hline Study & $\begin{array}{l}\text { Age } \\
\text { (yr) }\end{array}$ & Sex & Location & Level & Primary & Surgery & Radiotherapy & Recurrence \\
\hline Chou et al. [5] & 80 & Male & $\begin{array}{l}\text { Extramedullary, } \\
\text { intramedullary }\end{array}$ & Thoracal, T10 & + & Total & No & No \\
\hline Kashiwazaki et al. [8] & 31 & Male & $\begin{array}{l}\text { Extramedullary, } \\
\text { intramedullary }\end{array}$ & Thoracal, T4-6 & + & Total & No & No \\
\hline Liu et al. [9] & 32 & Female & Intramedullary & Thoracal,T5-6 & + & Total & Yes & No \\
\hline Liu et al. [9] & 24 & Male & Intramedullary & Cervical, C5-6 & + & Total & Yes & No \\
\hline Shirzadi et al. [10] & 27 & Female & $\begin{array}{l}\text { Intramedullary, } \\
\text { extramedullary }\end{array}$ & Thoracal, T7-8 & + & Total & No & No \\
\hline Our case 1 & 19 & Female & Intramedullary & Cervical, C1-2 & + & Total & No & No \\
\hline Our case 2 & 15 & Female & Intramedullary & Thoracal, T9-10 & + & Total & No & No \\
\hline
\end{tabular}

\section{Diagnostic features}

The tumors were located majorly in thoracic region (5/7, $71.4 \%$ ), between $\mathrm{T} 4$ to $\mathrm{T} 10$, and only two cases was in cervical region $(2 / 7,28.6 \%)$. The tumors were prone to localize at the dorsal side of the spinal cord. The cervical involvement was not level-specific, and they were seen at C1-2 and C5-7 levels.

MR images showed isointensity on T1-weighted images and high intensity on T2-weighted images. There were marked enhancements following contrast injections, in all tumors. The primary differential diagnosis was HPC followed by meningioma, neurogenic tumors, and metastases.

\section{Tumor characteristics}

All patients presented with the symptoms of a spinal cord tumor. There were no HPCs in other regions. However, the intramedullary tumor was extended to extramedullary area in $3 / 7$ cases $(42.8 \%)$. Although all tumors could be treated with total resection, firm consistency with indistinctive borders was described as an intraoperative finding for the tumor. In our series, tumor borders could be discriminated with microsurgical techniques in almost all around the tumor tissue, except in certain parts where the clear junction could not be distinguished.

Another discriminating observation during surgery was the prominent increase in vasculature, and in some reports, bleeding. However, this could not prevent the complete removal. The need for blood transfusion was neither reported nor specified in the articles. We experienced abundant bleeding, necessitating blood transfusion in our case number 2.

Histopathologically, the tumors were characteristically 
cellular with increased vascular structures and stag-horn vessels. None of the cases demonstrated anaplastic features (necrosis, increased mitotic activity, and pleomorphism) and high WHO grades. Immunohistochemical staining was specified in five cases, and all had positivity for CD34 staining and no reaction for S-100 proteins.

\section{Postoperative course and prognosis}

The tumors were followed without recurrences for a mean duration of 2.7 years ( 6 months to 6.7 years). All patients, except for two, reported with uneventful postoperative course. Regarding two cases with unfavorable outcomes, one patient was from the study by Chou et al. [5] and another patient was from our cases; they had gradual improvements in neurological findings. Two patients reported of receiving postoperative radiotherapy, and others did not report of having adjuvant therapies.

\section{Discussion}

HPCs are rare tumors in spinal region. Mena et al. [11] reviewed 94 cases, most of which were settled in the intracranial region. There were 13 cases in the spine, affecting cervical (6/13) and thoracic region (7/13). A previous literature review indicated a total of 52 spinal cases, mostly involving the extradural area [5]. Kruse [12] can be credited for reporting the first case of an intradural spinal HPC in 1961. Later on, the figure was updated to 11, in 2009 [5]. In the case of primary intramedullary involvement or invasion from an intradural lesion, the numbers are even less. There is paucity in the literature for the management and prognosis of intramedullary HPCs.

The differential diagnosis included HPCs, meningiomas, subpial schwannomas, metastatic tumors, and primary neurogenic tumors (ependymomas). In fact, these differentials, particularly HPC, can be insisted even during surgery, because neither clinical presentations nor radiological features are unique for intramedullary HPCs $[5,8]$. Patients can present with pain, sensory complaints, or even paralysis with respect to localizations. MR findings are not specific, and they are generally shown as isointense with spinal cord on T1-weighted images and hyperintense on T2-weighted. Regarding the enhancement following contrast injections, some reports advocate heterogeneous [9] while others advocate homogenous $[5,10]$ enhancements. Our experience demonstrated dense and homogenous post contrast enhancement in both of our intramedullary cases. Moreover, literature findings indicated that the presence of serpentine vessels, absence of calcifications [9], blurred borders between the tumor and spinal cord, and occasionally associated invasion of adjacent structures on MR imaging may advocate HPCs preoperatively. On the other hand, presence of dural involvement, dural tail sign, and erosion of adjacent structures (pedicles and neural foramen) can be observed, depending on the extent of involvement; and they are not specific [5,8-10]. Liu et al. [9] presented a series of 26 spinal HPCs in only four cases (15\%), and the HPC was correctly diagnosed. In fact, in two patients with intramedullary involvement, the preoperative diagnoses were not also accurate, and they were specified as HPC and ependymoma. They have stated about the difficulty in differentiating intraspinal HPC from other tumors in the same localizations [9]. Similarly, we have also favored HPC at the preoperative period and adhered to that during the surgery. This also supports the reported difficulty in radiological differential diagnosis of HPCs. The authors of this report practically accepted the misdiagnosing of HPC in the preoperative period as innocuous, for the sake of the patient, because similar vascularity pattern renders a similar surgical technique. However, the other preoperative diagnoses will have more disastrous consequences, if necessary preoperative workup is not performed as in our case number 2 .

HPCs, originating from capillary pericytes, have distinctive innate features. The relatively blurred surgical plane between the tumor and neuronal tissue was ascribed for tumoral infiltration, tracking the vessels into the deep neural structures [5].

Surgical strategies for these rare tumors have not been clear yet in the literature, due to small number of cases. Basically, two different techniques were described in the literature with or without preoperative embolization of the tumor [13]: piece-meal removal of the tumors with central debulking $[5,14]$ and meticulous dissection of the tumor from spinal cord without any internal decompression. Based on our experience with both techniques over two intramedullary cases, we favor the latter one because of the risk for aggressive bleeding and inadvertent risk of damaging spinal cord tissue, attributing to aforementioned infiltration pattern.

Benefit regarding extent of the resection was also questioned in the literature. Chou et al. [5] could not show 
any advantage of complete tumor removal on recurrence rates (53\%), irrespective of the localizations in intradural or extradural regions. On the contrary, Zweckberger et al. [15] stated about the importance of resection on tumor control. The series had five spinal cases (two cervical, one thoracic and two lumbar); among these, complete resection could be achieved, and there were no recurrences afterwards. Total removal was also advocated by other authors; independent from the tumor grade, complete removal of tumor was correlated with favorable outcomes [3]. Dufour et al. [16] presented four cases of spinal HPCs, and three of the cases were operated with complete resection. Chou et al. [5] supported no use of radiotherapy for preventing recurrences in neither extradural nor intradural HPCs. In our series, 2/7 patients received radiotherapy; none of the seven cases with total resections in our review demonstrated any recurrences, for the mean duration of 2.7 years (maximum, 6.7 years).

The authors support a surgical strategy aiming at the complete resection without any morbidity, because extent of the removal was correlated with long-term and recurrence-free survival. Liu et al. [9] classified 26 patients based on localization of tumors. They demonstrated that there was an inverse relationship between the rates of total tumor removal and the extent of tumor invasion out of the spinal canal. The extradural tumors embrace a more aggressive nature compared to intradural ones. A total of 19/26 patients (73\%) and 6/10 patients (60\%) of intradural HPC had recurrences. Their figures also supported our notion regarding different behavior of intramedullary HPCs, from both intradural and extradural counterparts, and none of the patients with intramedullary involvement had recurrence after surgery [9]. In some other reports, complete removal of the tumor was reported in $75 \%-82 \%$ of the intradural HPCs $[5,16]$. The rates were relatively higher compared to those with extraspinal involvements [10], and yet it was less than intramedullary HPCs. Similar findings were reported by Chou et al. [5] indicating that intradural HPCs are more prone to gross total resection compared to extradural HPCs. This was attributed to strong adhesions to dura, aggressive invasion of surrounding bony structures, and its high vascularity in addition to intradural HPCs' challenge which is invasion of neural structures. In the following series, our figure demonstrated that total tumor removal could be achieved in all intramedullary cases with favorable outcomes by using meticulous microsurgical technique. The rates of total tumor removal and favorable outcomes, the alleviated risk for recurrence, and the need for adjuvant therapies constitute the major differences of intramedullary HPCs vs. extramedullary HPCs.

\section{Conclusions}

Intramedullary HPCs are quite rare entities with favorable outcomes and benign temper with appropriate management strategies, compared to those located in other regions; total surgical resection seems to be the best and performable treatment option to avoid recurrences. Should the complete resection be achieved, no need for further treatment ensues. The follow-up should be tailored according to each patient.

\section{Conflict of Interest}

No potential conflict of interest relevant to this article was reported.

\section{References}

1. Guthrie BL, Ebersold MJ, Scheithauer BW, Shaw EG. Meningeal hemangiopericytoma: histopathological features, treatment, and long-term follow-up of 44 cases. Neurosurgery 1989;25:514-22.

2. Stout AP, Murray MR. Hemangiopericytoma: a vascular tumor featuring Zimmermann's pericytes. Ann Surg 1942;116:26-33.

3. Ramakrishna R, Rostomily R, Sekhar L, Rockhill J, Ferreira M. Hemangiopericytoma: radical resection remains the cornerstone of therapy. J Clin Neurosci 2014;21:612-5.

4. Louis DN, Ohgaki H, Wiestler OD, Cavenee WK. WHO Classification of tumors of the central nervous system. 4th ed. Lyon: International Agency for Research on Cancer; 2007.

5. Chou CW, Hsu SP, Lin SC, et al. Primary intradural hemangiopericytoma with intramedullary invasion. J Chin Med Assoc 2009;72:536-41.

6. Ujiie $\mathrm{H}$, Kubo O, Shimizu T, et al. Intramedullary hemangiopericytoma of the spinal cord; case report and review of the literature. No Shinkei Geka 1990;18:273-7.

7. Taniura S, Taniguchi M, Mizutani T, Takahashi H. Metastatic hemangiopericytoma to the cauda equina: 
a case report. Spine J 2007;7:371-3.

8. Kashiwazaki D, Hida K, Yano S, Seki T, Iwasaki Y. Subpial hemangiopericytoma with marked extramedullary growth: case report. Neurosurgery 2007;61:E1336-7.

9. Liu HG, Yang AC, Chen N, Yang J, Qiu XG, Zhang JG. Hemangiopericytomas in the spine: clinical features, classification, treatment, and long-term followup in 26 patients. Neurosurgery 2013;72:16-24.

10. Shirzadi A, Drazin D, Gates M, et al. Surgical management of primary spinal hemangiopericytomas: an institutional case series and review of the literature. Eur Spine J 2013;22 Suppl 3:S450-9.

11. Mena H, Ribas JL, Pezeshkpour GH, Cowan DN, Parisi JE. Hemangiopericytoma of the central nervous system: a review of 94 cases. Hum Pathol 1991;22:8491.

12. Kruse F Jr. Hemangiopericytoma of the meniges (angioblastic meningioma of Cushing and Eisenhardt): clinico-pathologic aspects and follow-up studies in 8 cases. Neurology 1961;11:771-7.

13. Ciappetta P, Celli P, Palma L, Mariottini A. Intraspinal hemangiopericytomas: report of two cases and review of the literature. Spine (Phila $\mathrm{Pa} 1976$ ) 1985;10:27-31.

14. Zhao Y, Zhao JZ. Clinical and pathological characteristics of primary intraspinal hemangiopericytoma and choice of treatment. Chin Med J (Engl) 2007;120:115-9.

15. Zweckberger K, Jung CS, Mueller W, Unterberg AW, Schick U. Hemangiopericytomas grade II are not benign tumors. Acta Neurochir (Wien) 2011;153:38594.

16. Dufour H, Metellus P, Fuentes S, et al. Meningeal hemangiopericytoma: a retrospective study of 21 patients with special review of postoperative external radiotherapy. Neurosurgery 2001;48:756-62. 